\title{
Polycystic Ovarian Syndrome and Early Pregnancy Loss
}

\author{
Authors \\ Dr Sheila Balakrishnan ${ }^{1}$, Dr Benu Majeed .K.C. ${ }^{2}$ \\ ${ }^{1}$ Professor, Dept. of Obstetrics and Gynaecology GMCT \\ ${ }^{2}$ Junior Resident, Dept. of Obstetrics and Gynaecology GMCT
}

\begin{abstract}
Background: Patients with Polycystic ovarian syndrome is found to have an increased risk of spontaneous abortions. This study aims to determine the effect of PCOS status on the risk of early pregnancy loss in a cohort of pregnant women where the PCOS status was determined by standard criteria.

Materials and Methods: This is a prospective cohort study conducted over a period of one year. Antenatal women attended the obstetrics OP department SAT hospital, Govt medical college, Trivandrum soon after pregnancy was confirmed and diagnosed to have polycystic ovarian syndrome according to Rotterdam criteria were included in the study. For comparison we selected the same number of pregnant women, those attended in our antenatal OP department soon after pregnancy was confirmed and they had regular cycles and in ultrasound scan showed normal ovaries and they didn't had any features of PCOS. Student t-test or Chi-square tests were used to test the difference between PCOS and non PCOS group.

Results: The incidence of first trimester spontaneous abortion was $29.7 \%$ in the study group. The chance of recurrent pregnancy loss was found to be increased in PCOS group compared to control group, but statistically not significant $(p=0.354)$. Occurrence of missed abortion in PCOS pregnancy patients is high (17.8\%) compared to non PCOS pregnancy patients.

Conclusion: PCOS women are at increased risk of early pregnancy loss. Several mechanisms underlying the increased risk of early pregnancy loss in women with PCOS have been proposed.

Keywords: PCOS, early pregnancy loss, BMI.
\end{abstract}

\section{Introduction}

Polycystic ovary syndrome (PCOS) is a heterogeneous endocrine disorder that affects several body systems and leads to reproductive and metabolic complications ${ }^{[1]}$. The increased metabolic risk might be related to obesity as well as to genetic and environmental factors ${ }^{[2]}$. Prevalence of PCOS in India is $22.5 \%$ (by Rotterdam criteria) and $10.7 \%$ (by Androgen excess society criteria) ${ }^{[3]}$. Globally its prevalence ranges from $2.2 \%$ to $26 \%$ Most studies in India shows prevalence of $9.13 \%$ to $36 \%^{[4]}$. The condition is characterised by oligo-ovulation or anovulation, hyperandrogenism, and polycystic ovaries. It is frequently associated with insulin resistance and obesity. The National Institutes of Health (NIH) recommended in 1990 to use hyperandrogenism and ovulatory dysfunction as diagnostic criteria and that ultrasound should not form part of the process. The currently most 
widely used 2003 ESHRE/ASRM (Rotterdam) definition emphasizes the importance of ultrasound as a diagnostic tool.

Accordingly, PCOS is diagnosed when two of the following criteria are present - oligoovulation or anovulation, clinical or biochemical signs of hyperandrogenism, polycystic ovaries on ultrasound scan defined as the presence of 12 or more follicles in each ovary and an increased ovarian volume and exclusion of other etiologies. PCOS is associated with $30-50 \%$ rate of pregnancy loss. About $36 \%-82 \%$ of women with recurrent pregnancy loss are reported to have PCOS or anatomically polycystic ovaries. Therefore, the presence of anatomically polycystic ovaries has been postulated to contribute to miscarriage in PCOS, either through ovarian androgen hypersecretion or LH hypersecretion. Obesity has also been implicated as a possible risk factor for early pregnancy loss and recurrent miscarriages in PCOS. Although obesity has been shown to be a risk factor for pregnancy loss in PCOS, it remains unclear if obesity itself or an obesity associated comorbidity, such as insulin resistance, is responsible for this phenomenon.

Increased activity of Plasminogen Activator Inhibitor1 [PAI-1], major inhibitor of fibrinolysis has been reported to promote RPL in normal women. Elevated PAI-1 activity is an independent risk factor for miscarriage in women with PCOS. : Deficient secretion of endometrial proteins may contribute to miscarriage in PCOS. Glycodelin (previously known as PP14) and IGF binding protein-1 (IGFBP-1) are two proteins secreted by the endometrium that appear to play important roles in endometrial receptivity during implantation and early pregnancy. Women with PCOS have been shown to have both low serum glycodelin concentrations and serum IGFBP-1 concentrations in pregnancy. Metformin increases concentration of IGFBP-1 \& Glycodelin ${ }^{[5]}$. Studies suggested that women with hypersecretion of luteinizing hormone ( $\mathrm{LH})$, a frequent feature of PCOS, are at increased risk for miscarriage after either spontaneous or assisted conception ${ }^{[6]}$.
Elevated androgen levels (testosterone and androstenedione) have been postulated to play a role in miscarriage in PCOS.

The reproductive issues with PCOS are manifold starting with anovulatory cycles leading to subfertility. Post conception, PCOS women are at increased risk for early pregnancy loss (EPL). After having successfully passed the first trimester, they commonly encounter later pregnancy complications like gestational diabetes mellitus (GDM), pregnancy-induced hypertension $(\mathrm{PIH})$, preeclampsia, preterm delivery, and birth of small for gestational age (SGA) infant.

\section{Materials and Methods}

This is a prospective cohort study conducted in the department of Obstetrics and Gynaecology, Government Medical College, Trivandrum. Antenatal women who attended the obstetrics OP department SAT hospital, Government Medical College, Trivandrum soon after pregnancy was confirmed and diagnosed to have polycystic ovarian syndrome according to Rotterdam criteria formed the study group. For comparison we selected same number of pregnant women, those who attended in our antenatal OP department soon after confirming pregnancy and had regular cycles and ultrasound scan showed normal ovaries and did not had any features of PCOS. Data was collected using a pre-structured questionnaire. $\mathrm{T}$ test and Chi square test was used to analyse the data.

\section{Results}

Out of 118 PCOS patients in the study group, $57.6 \%$ conceived by treatment for infertility, $42.4 \%$ by spontaneous conception. Out of 118 non PCOS patients $91.5 \%$ conceived spontaneously without any treatment .So Statistically significant number of PCOS patients $(\mathrm{p}<0.001)$ was conceived following treatment for infertility. In pregnant women with PCOS $22.9 \%$ was overweight and $10.2 \%$ are obese, Hence, high BMI is statistically significant in PCOS with $\mathrm{p}<0.001$. 
Table 1 Distribution of BMI in PCOS and non PCOS patients

\begin{tabular}{|l|c|c|c|c|c|c|}
\hline \multirow{2}{*}{} & \multicolumn{5}{|c|}{ BMI } & \multirow{2}{*}{ Total } \\
\cline { 2 - 7 } & & Under weight & Normal & Over weight & Obese & \\
\hline \multirow{3}{*}{ PCOS } & $\mathrm{N}$ & 11 & 68 & 27 & 12 & 118 \\
\cline { 2 - 7 } & $\%$ & 9.3 & 57.6 & 22.9 & 10.2 & 100 \\
\hline No & $\mathrm{N}$ & 58 & 57 & 2 & 1 & 100 \\
\cline { 2 - 7 } & $\%$ & 49.2 & 48.3 & 1.7 & 0.8 & 100 \\
\hline \multirow{3}{*}{ Total } & $\mathrm{N}$ & 69 & 125 & 29 & 13 & 236 \\
\cline { 2 - 7 } & $\%$ & 29.2 & 53.0 & 12.3 & 5.5 & 100 \\
\hline
\end{tabular}

$\chi^{2}=53.281 . \quad \mathrm{df}=3 \quad \mathrm{p}<.001$

First trimester pregnancy loss is the pregnancy loss which occurred at gestational age less than 12 weeks of period of amenorrhea. It can be missed abortion, anembryonic pregnancy, ectopic pregnancy. This tables shows that the risk of first trimester loss in PCOS patients is more than in non PCOS patients. In the study group of 118 PCOS patients, $29.7 \%$ had first trimester loss where in non PCOS patients it was $6.8 \%$ only. So the risk of first trimester loss is significantly high in PCOS cohort with relative risk $(95 \% \mathrm{CI})=$ 4.375 (2.120-9.028) and $p<0.001$

Table 2 PCOS and early pregnancy loss

\begin{tabular}{|c|c|c|c|c|c|}
\hline \multirow{2}{*}{\multicolumn{3}{|c|}{. }} & \multicolumn{2}{|c|}{ 1st trimester loss } & \multirow{2}{*}{ Total } \\
\hline & & & Yès & No & \\
\hline \multirow{5}{*}{ Category } & \multirow{2}{*}{$\begin{array}{c}\cdot \\
\mathrm{PCOS}\end{array}$} & $\mathrm{N}$ & 35 & 83 & 118 \\
\hline & & $\%$ & 29.7 & 70.3 & 100.0 \\
\hline & \multirow{3}{*}{ No PCOS } & $\mathrm{N}$ & 8 & 110 & 100 \\
\hline & & & & & \\
\hline & & $\%$ & 6.8 & 93.2 & 118 \\
\hline
\end{tabular}

Relative Risk $(95 \%$ CI $)=4.200(1.638-10.766)$

Occurrence of missed abortion in PCOS pregnancy patients is high (17.8\%) compared risk of 4.200. Hence, missed Abortion in to non PCOS pregnancy patients with relative PCOS is statistically significance $(\mathrm{p}=0.001)$. 
Table 3 Missed abortion in PCOS

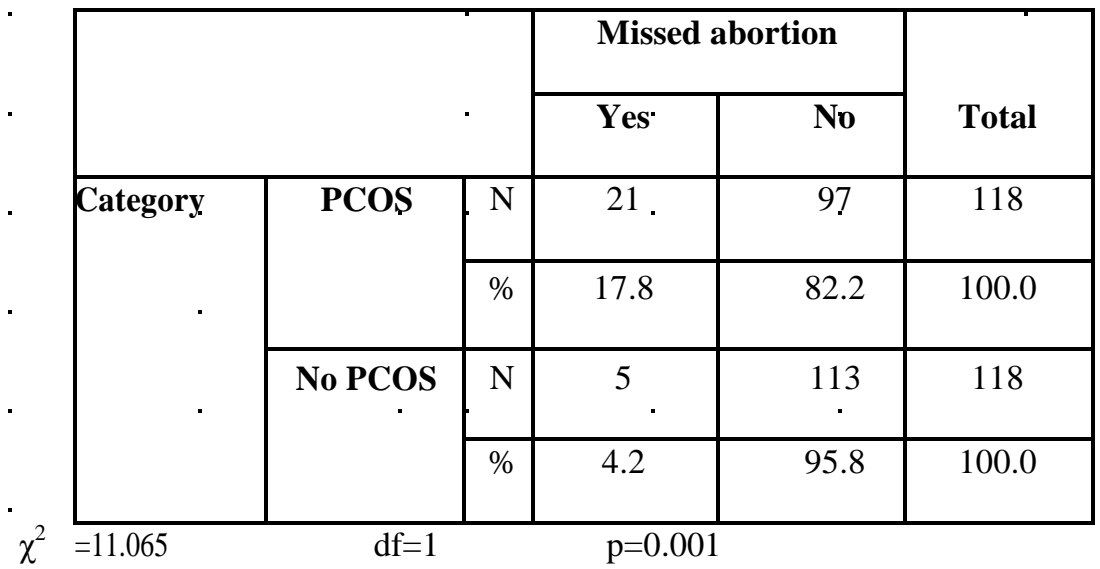

Relative Risk $(95 \%$ CI $)=4.200(1.638-10.766)$

Occurence of anembryonic Pregnancy is more in PCOS than non PCOS pregnancies

Relative Risk $(95 \% \quad \mathrm{CI})=0.932 \quad(0.888-$ 0.979).

with statistical significance $(\mathrm{p}=0.004)$.

Table 4 PCOS and anembryonic pregnancy

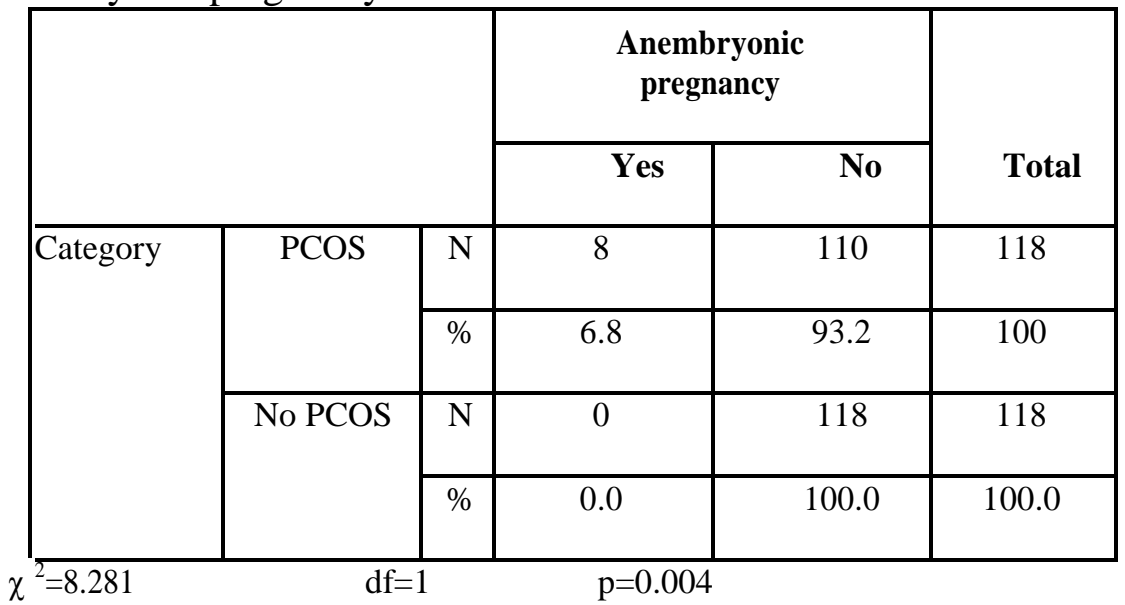

The chance of recurrent pregnancy loss was found to be increased in PCOS group

compared to control group, but statistically not significant $(\mathrm{p}=0.354)$.

Table 5 Recurrent pregnancy loss and PCOS

\begin{tabular}{|c|c|c|c|c|c|}
\hline & & & & RPL & \\
\hline & & & Yes & No & \\
\hline & & $\mathrm{N}$ & 7 & 111 & 118 \\
\hline & PCO & $\%$ & 5.9 & 94.0 & 100.0 \\
\hline Category & & $\mathrm{N}$ & 4 & 114 & 118 \\
\hline & No PCUS & $\%$ & 3.4 & 96.6 & 100.0 \\
\hline
\end{tabular}




\section{Discussion}

Polycystic ovary syndrome is a common endocrine disorder affecting $9.13 \%$ to $36 \%$ of women in India. It is characterised by oligoovulation or anovulation, hyperandrogenism and polycystic ovarian morphology and may be associated with insulin resistance and obesity. In this study $51.7 \%$ of the PCOS group were under $26-30 \mathrm{yrs}$ of age. $57.6 \%$ of PCOS women conceived by taking treatment for infertility. Women with polycystic ovary syndrome can conceive spontaneously but with a delayed fertile window since there is a tendency of regular menstrual cycles with advancing age. In the present study we found that women with polycystic ovary syndrome were slightly older than women without polycystic ovary syndrome.

More than $50 \%$ of PCOS patients are obese. Stephano Palombo et al suggested that the co morbidity most closely related to PCOS is obesity. Obesity increased the risk of miscarriage, hypertension, diabetes and increased chance of caesarian section in PCOS pregnancies.. According to present study obesity is increased in women with PCOS with $\mathrm{p}<0.001$.

It is still debated whether women in PCOS have increased risk of miscarriage compared with women without PCOS. R. Humbarg et al conducted a study about the influence of serum L.H concentration on ovulation conception and early pregnancy loss in 54 patients with PCOS. That study indicates that raised basal LH concentration and raised LH concentration at the time of maximum follicular growth significantly interfere with conception and may be causal factor for early pregnancy loss. In the study conducted with 54 patients with PCOS early pregnancy loss happen in 9 cases $(\mathrm{p}=0.01)$. Recently a large Australian study demonstrated that miscarriage rate was more frequent in women with PCOS than in control (20\% vs $15 \%, \mathrm{p}=0.003)$. Although PCOS was not an independent risk factor for pregnancy loss but miscarriage rate was strongly influenced by BMI (Joham et al 2014 a ).First trimester pregnancy loss is the pregnancy loss which occurred at gestational age less than 12 weeks of period of amenorrhea. It can be missed abortion, anembryonic pregnancy or ectopic pregnancy. In the current study of 118 PCOS patients, $29.7 \%$ had first trimester loss where in non PCOS patients it was $6.8 \%$ only. So the risk of first trimester loss is significantly high in PCOS cohort with relative risk $(95 \% \mathrm{CI})=4.375(2.120$ 9.028) and $\mathrm{p}<0.001$. But in PCOS group second trimester loss occurred in $7.2 \%$. In study cohort, $6.8 \%$ reported to have Anembryonic pregnancy, $17.8 \%$ cases missed abortion, $2.5 \%$ cases of ectopic pregnancy.

\section{Conclusions}

Majority of women with PCOS come under age $>25$ years and $57.6 \%$ conceived following infertility treatment. About $35 \%$ of the patients had BMI $>25$. According to this study there was a significantly increased risk of first trimester pregnancy loss. Both missed abortion and anembryonic pregnancy were increased in pregnant women with PCOS.

\section{References}

1 Norman RJ, Noakes M, Wu R, Davies MJ, Moran L and Wang JX(2004) Improving reproductive performance in overweight/ obese women with effective weight management. Hum Reprod Update 10,267-280

2 Ehrmann DA, Liljenquist DR, Kasza K, Azziz R, Legro RS, Ghazzi MN PCOS/ Troglitazone Study Group. Prevalence and predictors of the metabolic syndrome in women with polycystic ovary syndrome. J Clin Endocrinol Metab2006;91:48-53.

3 Indian journal of Endocrinology and Metabolism; june 2014 ;Cross sectional study of polycustic ovarian syndrome among adolesent and young girls in Mumbai

4 Nidhi R Padmalatha V, Nagrathan R , Amrithanshu R, Prevalence of polycystic 
ovarian syndrome in india adolescents. $\mathrm{J}$ Pediatric Adolesc Gynecol 2011;24:233-7.

5 Julkunen M, Koistinen R, Suikkari AM, Seppala M, Janne OA, 1990 Identification by hybridization histochemistry of human endometrial cells expressing mRNAs encoding a uterine beta-lactoglobulin homologue and insulin-like growth factorbinding protein-1. Mol Endocrinol 4: 700707.

6 Homburg R, Armar NA, Eshel A, Adams J, Jacobs HS, 1998 Influence of serum luteinising hormone concentrations on ovulation, conception, and early pregnancy loss in polycystic ovary syndrome. BMJ 297: 1024-1026. 